\title{
Réplique de Sheldon et Judith Godfrey au compte rendu de leur ouvrage Search Out the Land: the Jews and the Growth of Equality in British Colonial America, 1740-1867, par Donald Fyson.
}

\section{Sheldon Godfrey et Judith Godfrey}

Volume 52, numéro 3, hiver 1999

URI : https://id.erudit.org/iderudit/005448ar

DOI : https://doi.org/10.7202/005448ar

Aller au sommaire du numéro

Éditeur(s)

Institut d'histoire de l'Amérique française

ISSN

0035-2357 (imprimé)

1492-1383 (numérique)

Découvrir la revue

Citer cet article

Godfrey, S. \& Godfrey, J. (1999). Réplique de Sheldon et Judith Godfrey au compte rendu de leur ouvrage Search Out the Land: the Jews and the Growth of Equality in British Colonial America, 1740-1867, par Donald Fyson. Revue d'histoire de l'Amérique française, 52(3), 434-437.

https://doi.org/10.7202/005448ar d'utilisation que vous pouvez consulter en ligne. 


\section{COMPTE RENDU}

Réplique de Sheldon et Judith Godfrey au compte rendu de leur ouvrage Search Out the Land: the Jews and the Growth of Equality in British Colonial America, 1740-1867, par Donald Fyson.

Dans son compte rendu de Search Out the Land: the Jews and the Growth of Equality in British Colonial America, 1740-1867 (Montréal, McGill-Queen's University Press, 1995), paru dans le numéro du printemps 1998 de votre Revue, Donald Fyson fait erreur lorsqu'il suggère que notre «hypothèse fondamentale» est que seuls «les Juifs (et autres groupes non anglicans) ont connu en Amérique du Nord britannique une égalité juridique et politique sans pareille», au même titre que la classe dirigeante anglicane, ce qui laisse supposer que cette égalité était refusée aux catholiques canadiens-français.

Les Juifs du Canada ont effectivement connu une égalité juridique et politique sans pareille entre 1760 et 1840. Par contre, loin de dominer la vie politique des deux Canadas, comme on le suggère dans le compte rendu, les protestants dissidents n'ont pas toujours connu les mêmes droits pendant cette période. Fait peut-être encore plus intéressant pour les lecteurs francophones, nous avons découvert que les Canadiens français ont joui d'une égalité juridique sans précédent à partir de l'Acte de Québec en 1774 et que ce nouveau statut a entraîné un mouvement en faveur de l'égalité de toutes les autres minorités religieuses dans les deux Canadas, formant ainsi la base d'une nation extraordinaire. Dans les autres colonies de l'Amérique du Nord britannique, aux États-Unis indépendants depuis peu et en Grande-Bretagne, les droits civiques et politiques des catholiques étaient beaucoup plus restreints qu'au Canada.

Nos résultats sont fondés en grande partie sur de nouvelles sources qui n'ont été analysées dans aucun ouvrage déjà paru. Notre étude poussée des structures juridiques des colonies nous a obligés à aller au-delà des sources traditionnelles d'information afin d'établir les critères qui définissent l'exercice plein et entier des droits civiques et politiques, tels l'obtention d'un poste gouvernemental. Nous avons découvert que durant le siècle qui a précédé l'introduction du gouvernement responsable, alors que le rôle du gouverneur était de première importance, les instructions secrètes au gouverneur comprenaient des clauses qui accordaient l'exercice plein et entier des droits aux loyaux citoyens qui prononceraient les déclarations et serments prescrits et qui créaient des «incapacités» pour d'autres citoyens, selon le texte des serments d'office.

Déjà en 1775 , soit seulement quinze ans après la Conquête, les instructions au gouverneur de Québec accroissaient considérablement les droits accordés à

RHAF, vol. 52, n 3, hiver 1999 
l'origine aux Canadiens français par l'Acte de Québec. Loin d'être «une exemption spéciale» de courte durée, comme le suggère Donald Fyson, le nouveau traitement réservé aux Canadiens français équivalait à un nouveau statut d'égalité juridique permanent, sans précédent, qui a été confirmé par la suite dans les instructions aux gouverneurs qui ont suivi. L'Acte de Québec permettait aux catholiques de substituer un serment œcuménique aux Serments d'État qui offensaient les catholiques et qui auparavant étaient requis pour obtenir certains postes. Les instructions données au gouverneur Guy Carleton l'année suivante allaient même plus loin en exemptant tout «Canadien professant la religion de l'Église de Rome» de la Déclaration d'adhésion à l'Église d'Angleterre imposée par le Test Act, même si elle était toujours requise des fidèles de toutes les autres religions (à l'exception des Juifs) pour pouvoir siéger au Conseil du gouverneur.

Les autres minorités religieuses ne bénéficiaient pas de conditions aussi avantageuses que les catholiques canadiens-français et les Juifs. Contrairement à ce qui se faisait pour les Canadiens français, les incapacités résultant des instructions du gouverneur étaient toujours appliquées à tous les autres catholiques du Québec, ce qui les empêchait d'occuper de hautes fonctions entre 1775 et 1791. Ces incapacités s'appliquèrent à tous les protestants dissidents de 1791 à 1840. Bien que les protestants dissidents aient eu le droit d'être élus à l'Assemblée et de détenir des postes gouvernementaux mineurs (comme ceux de magistrat et d'officier dans la milice) pendant toute cette période, nous n'avons trouvé aucun exemple d'un protestant dissident dans le Bas-Canada entre 1791 et 1838 qui aurait occupé les fonctions de membre du Conseil exécutif ou du Conseil législatif, de juge d'une haute cour ou de tout autre poste d'importance que les Canadiens français occupaient librement depuis 1775. Cela contredit l'affirmation de l'auteur du compte rendu qui prétend que «au moins au Bas-Canada, des protestants non anglicans, notamment des presbytériens, détiennent de nombreux postes» après 1791 .

Enfin, nous avons trouvé que les droits civiques et politiques des catholiques canadiens-français étaient partout beaucoup plus restreints qu'au Québec ou dans les deux Canadas. En 1775, aucune des treize colonies américaines ne permettait aux catholiques d'exercer de droits civiques ou politiques; les colonies maritimes de l'Amérique du Nord britannique ne permettaient qu'aux seuls anglicans d'obtenir des postes gouvernementaux ou de siéger à l'Assemblée. L'égalité juridique des catholiques au Québec a précédé de plus de cinquante ans celle des catholiques en Grande-Bretagne.

Les Canadiens français ont disposé de majorités à l'Assemblée depuis la première Assemblée du Bas-Canada en 1792 et ont continué à avoir accès aux plus hautes fonctions, faisant ainsi du Canada un pays d'un genre différent où la loi obligeait la classe dirigeante anglicane à accorder aux autres les mêmes droits qu'à elle-même. La conclusion de Search Out the Land est que ce fut le traitement réservé aux Canadiens français par les Britanniques qui a fait du Canada 
«une nouvelle nation où l'égalité, plutôt que la tolérance, constituait la philosophie dominante».

\section{Réponse de Donald Fyson à la réplique de Sheldon et Judith Godfrey}

La réplique de Sheldon et Judith Godfrey me rend encore moins confiant en leurs conclusions. D'une part, ils interprètent mal mon compte rendu et me font dire des choses que je n'ai jamais dites. D'autre part, ils répètent des inexactitudes que je n'avais signalées dans mon compte rendu qu'après une vérification empirique.

D'abord, je n'ai ni dit ni laissé entendre que les Canadiens français catholiques ne jouissaient pas d'une égalité juridique et politique formelle après 1775 . Aucun historien sérieux du Québec ne ferait une telle affirmation. Le débat sur les statuts relatifs des francophones et des anglophones se passe sur d'autres plans: le pouvoir politique réel, la place dans l'économie, etc. Par ailleurs, il est évident que les catholiques jouissent d'une «exemption spéciale» à partir de 1775 pour leur donner accès aux postes gouvernementaux, car ce n'est pas la norme dans l'Empire britannique; comme je l'ai dit, cette exemption démarque le Québec de la plupart des autres colonies britanniques. Par contre, mon compte rendu ne dit nulle part que cette exemption est de «courte durée», car ce n'est pas le cas.

Ensuite, en ce qui concerne les protestants non anglicans, je n'ai jamais dit ni laissé entendre qu'ils aient pu «dominer la vie politique des deux Canadas». Affirmer une telle chose serait, encore une fois, parfaitement ridicule. Toutefois, sur l'exclusion de ces protestants des hauts postes gouvernementaux entre 1791 et 1838 , les Godfrey ont, tout simplement, tort. Le juge en chef William Smith, en poste avant et après 1791, est un presbytérien convaincu. Plusieurs membres du Conseil législatif du Bas-Canada sont des presbytériens: William McGillivray, Peter McGill, Horatio Gates, John Forsyth... Le gouverneur Dalhousie luimême est un presbytérien. Quels sont donc les postes réservés aux anglicans et aux catholiques?

Pour ce qui est des statuts différents des catholiques francophones et non francophones avant 1791, les Godfrey ont encore tort. L'Acte de Québec exempte très clairement tous les catholiques résidents au Québec des serments anticatholiques, peu importe leur ethnie: «[...] no Person professing the Religion of the Church of Rome, and residing in the said Province, shall be obliged to take the Oath [...]» (s.7). Si les instructions secrètes des gouverneurs étaient allées à l'encontre d'un acte du Parlement britannique, c'est ce dernier qui aurait primé (car, je le répète, les instructions aux gouverneurs n'ont aucune valeur constitutionnelle).

Je souligne aussi que la réplique des Godfrey reste muette quant aux autres erreurs importantes que j'ai relevées dans mon compte rendu (la notion de la nonapplication du Test Act avant 1791, le statut «protestant» des Juifs, etc.). Je prends la liberté de corriger certaines autres inexactitudes touchant les questions juridiques et constitutionnelles: la situation des lois civiles françaises dans la 
décennie suivant la Conquête est beaucoup plus complexe qu'une simple rétention générale (p. 96); il n'y pas un «civil code» au Québec à partir de 1774 (p. 120, 174); le gouverneur n'est pas capable d'importer le droit britannique dans la colonie de par sa seule proclamation (p. 120); la décision d'un gouverneur d'octroyer un poste n'est pas une «loi» (p. 126); l'«établissement» de l'Église anglicane entre 1763 et 1791 est loin d'être acquis (p. 131); les catholiques du Québec ne peuvent pas accéder à la plupart des postes gouvernementaux et juridiques à partir de 1760 (p. 132, 134, 135); plusieurs juges de la colonie d'avant 1790 ont une formation juridique (p. 140); il y a certainement des officiers au Bas-Canada chargés des archives gouvernementales (p. 174). Pour corriger leur «étude poussée des structures juridiques de la colonie», je suggère donc aux auteurs une lecture des ouvrages classiques de Burt, Neatby, Morel, auxquels on peut ajouter les études plus récentes de Hay, Fecteau, Kolish, etc.

Enfin, sur leur conclusion d'un Canada fondé sur l'égalité, démontré par le traitement accordé aux Canadiens français, je n'ai que deux commentaires. D'une part, il me semblait que le livre portait sur les Juifs plutôt que sur les Canadiens français, d'où le peu de mention de ces derniers dans mon compte rendu. D'autre part, même en laissant de côté la question épineuse des Canadiens français (qui est d'ailleurs beaucoup plus complexe qu'un débat simpliste entre égalité et oppression), le Canada, comme la plupart des nations occidentales, est fondé sur l'inégalité: entre hommes et femmes, entre Européens et autres, entre riches et pauvres, sans pour autant exclure d'autres manifestations d'inégalité. Ces inégalités sont le reflet des valeurs fondamentales des sociétés de l'époque, qui sont foncièrement inégalitaires. Une petite lecture de l'histoire des autochtones, des noirs, des femmes, des travailleurs, des immigrants, nous permet de rejeter aisément la théorie qu'en 1867, «British North America was already advanced and enlightened in its attempts to ensure that all minorities were legally equal» (p. xxiii). C'est une vision foncièrement rose de l'histoire du Canada. 